\title{
Abdominopelvic Actinomycosis Mimicking Peritoneal Carcinomatosis: A Case Report
}

\author{
Sungjin Kim ${ }^{1}$, Sung Il Kang ${ }^{1}$, Sohyun Kim ${ }^{1}$, Min Hye Jang ${ }^{2}$, Jae Hwang Kim ${ }^{1}$ \\ Departments of ${ }^{1}$ Surgery and ${ }^{2}$ Pathology, Yeungnam University Medical Center, Daegu, Korea
}

Actinomycosis is a rare chronic bacterial infection primarily caused by Actinomyces israelii. A 47-year-old woman presented to our clinic with a 1-week history of lower abdominal pain. Preoperative imaging studies revealed multiple peritoneal and pelvic masses suggestive of malignancy. The primary tumor could not be identified despite further endoscopic and gynecological evaluation. On exploration for tissue confirmation, excisional biopsies from multiple masses were performed because complete excision was not possible. Histopathological examination confirmed actinomycosis with multiple abscesses, and the patient was treated with antibiotics. We present a case of disseminated peritoneal actinomycosis that mimicked malignant peritoneal carcinomatosis on imaging studies.

Keywords: Actinomycosis; Carcinomatosis; Intrauterine devices

\section{INTRODUCTION}

Actinomycosis is a rare, chronic or subacute, bacterial infection primarily caused by Actinomyces israelii, an anaerobic gram-positive or microaerophilic/capnophilic organism belonging to the Actinomyces genus [1]. The clinical presentation of actinomycosis can mimic that of malignancies, and the pre- and intraoperative diagnosis is challenging owing to the nonspecific clinical findings observed in infected patients [2]. We report a rare case of abdominopelvic actinomycosis of unknown primary origin that mimicked peritoneal carcinomatosis.

\section{CASE REPORT}

A 47-year-old woman was referred to our clinic with a 1-week history of lower abdominal pain and a palpable mass on abdomi-

Received: October 1, 2019 - Accepted: November 7, 2019

Correspondence to: Sung II Kang, M.D.

Department of Surgery, Yeungnam University Medical Center, Yeungnam University College of Medicine, 170 Hyeonchung-ro, Nam-gu, Daegu 42415, Korea

Tel: +82-53-620-3580, Fax: +82-31-787-4055

E-mail: sungiry@naver.com

ORCID: https://orcid.org/0000-0002-4751-5779

(C) 2020 The Korean Society of Coloproctology

This is an open-access article distributed under the terms of the Creative Commons Attribution NonCommercial License (https://creativecommons.org/licenses/by-nc/4.0) which permits unrestricted noncommercial use, distribution, and reproduction in any medium, provided the original work is properly cited. nal examination. She denied a history of abdominal operations, but she reported intrauterine device (IUD) implantation 5 years prior to presentation. She had been diagnosed with a uterine myoma and a perirectal mass by pelvic magnetic resonance imaging at a local medical center. The patient's vital signs were within the normal range. Abdominal examination revealed a hard mass in the left lower quadrant (LLQ) of the abdomen with no acute peritoneal signs. Gynecological examination revealed no findings suggestive of gynecological malignancy; however, her serum cancer antigen (CA) 125 level was elevated (89 U/mL). Other blood tests were negative for leukocytosis or C-reactive protein elevation, which was not suggestive of systemic inflammation. Serum levels of carcinoembryonic antigen and CA19-9 were also within the normal range.

The IUD was removed by a gynecologist. Contrast-enhanced abdominopelvic computed tomography (CT) revealed a huge enhancing mass connected to the greater omentum in the left lower abdomen and a perirectal mass connected to the uterus, suggestive of peritoneal carcinomatosis (Fig. 1). Positron-emission tomography performed for diagnosis of the primary malignancy revealed a suspected carcinomatous lesion in the LLQ in addition to multiple masses with fluorodeoxyglucose uptake near the right lower quadrant and the perirectal area (Fig. 2). Endoscopic examination for assessment of alimentary tract malignancy did not reveal any lesions suggestive of malignancy. Therefore, surgical exploration was performed for tissue confirmation.

Laparoscopic exploration was impossible because of a frozen 


\section{Annals of Abdominopelvic Actinomycosis Mimicking Peritoneal Carcinomatosis: A Case Report}

\section{Coloproctology sungjin Kim, et al.}

abdomen secondary to the omental mass. Therefore, a median laparotomy was performed and revealed a firm mass which was strongly adherent to the left lower parietal peritoneum and connected to the greater omentum around it. The mass projected from the posterior sheath of the rectus abdominis, and histopathological examination demonstrated a focal nodular fibrotic lesion with central necrosis (Fig. 3A, B). A similar mass protruded from the right lower abdominal wall but did not involve the abdominal visceral peritoneum (Fig. 3C, D). The appendix in the vicinity of the mass appeared normal. Another firm mass was identified attached to the uterus on the pouch of Douglas which did not invade the rectal wall (Fig. 3E, F). Frozen section biopsies from multiple sites revealed chronic inflammation with atypical cells, so malignancy could not be excluded. Finally, the operation was
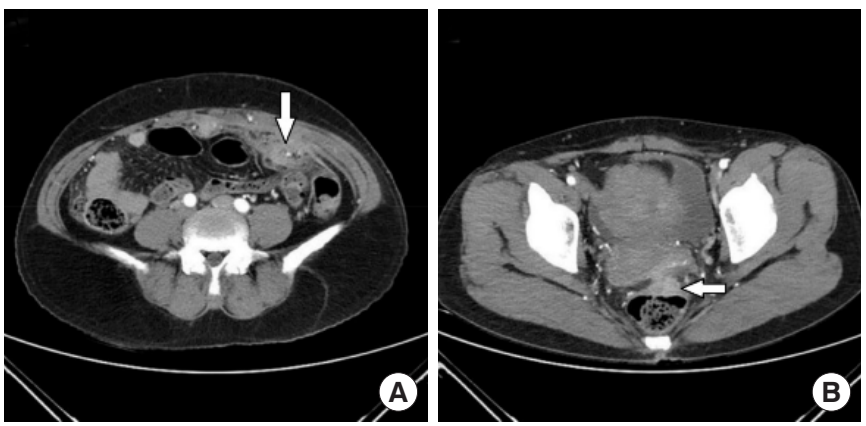

Fig. 1. (A) Contrast-enhanced abdominal computed tomography scan showed a huge enhancing mass (arrow) connected to the greater omentum. (B) A perirectal mass (arrow) was also visualized. abandoned because significant adhesions between the major vessels, intra-abdominal organs and the abdominal wall prevented complete resection.

The patient's postoperative recovery was uneventful. The final histopathological findings were consistent with a diagnosis of ac-
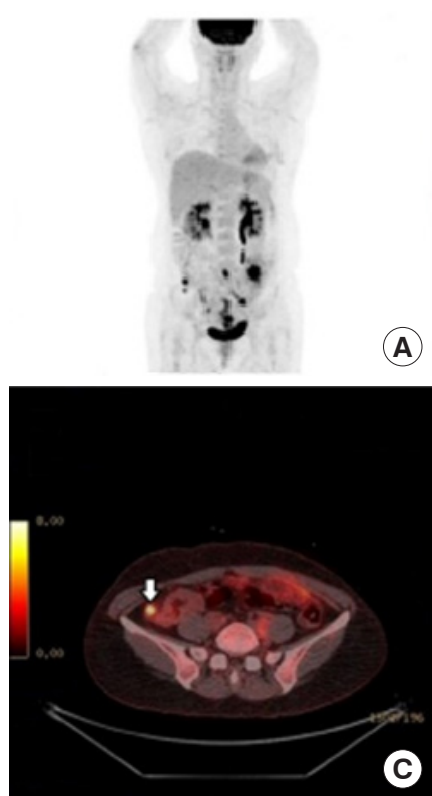

A
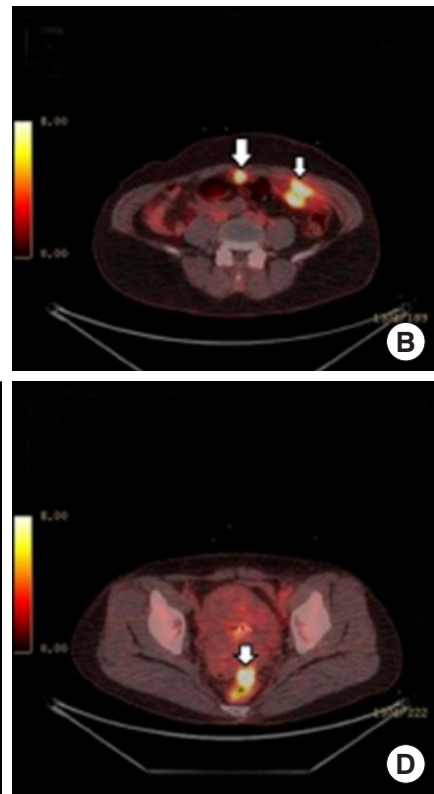

Fig. 2. Positron-emission tomography scan revealed a suspected carcinomatous lesion in the left lower quadrant and multiple masses with positive fluorodeoxyglucose uptake.
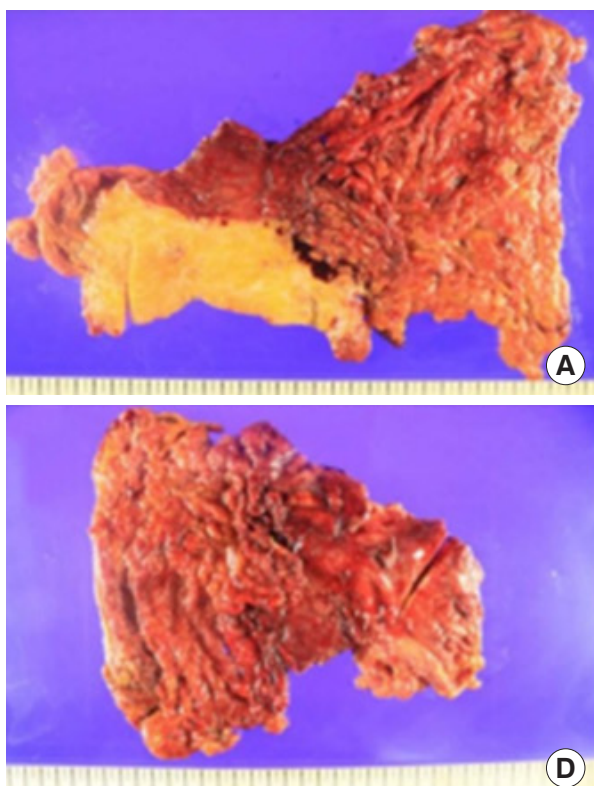
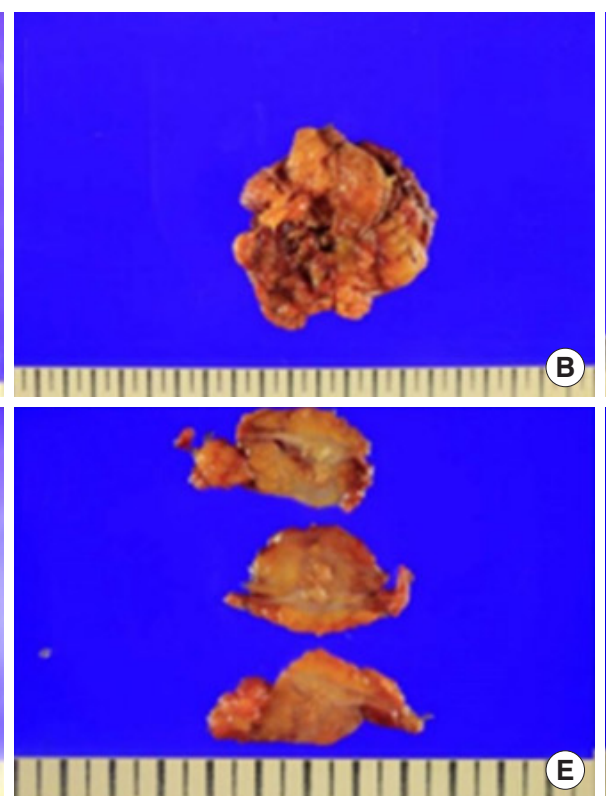
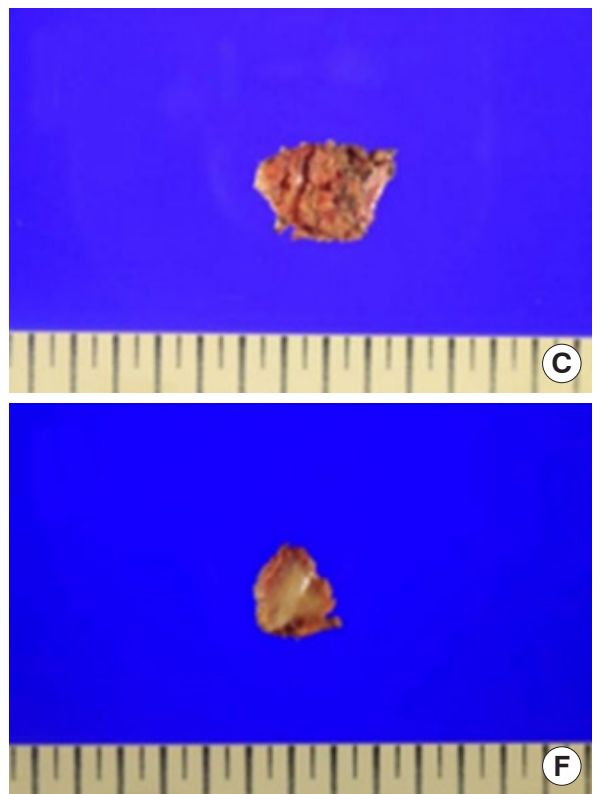

Fig. 3. Histopathological examination of the gross findings. (A, B) A mass attached to the left lower parietal peritoneum and connected to the greater omentum was observed. (C, D) The mass protruded from the right lower abdominal wall. (E, F) A perirectal mass was also visualized. 


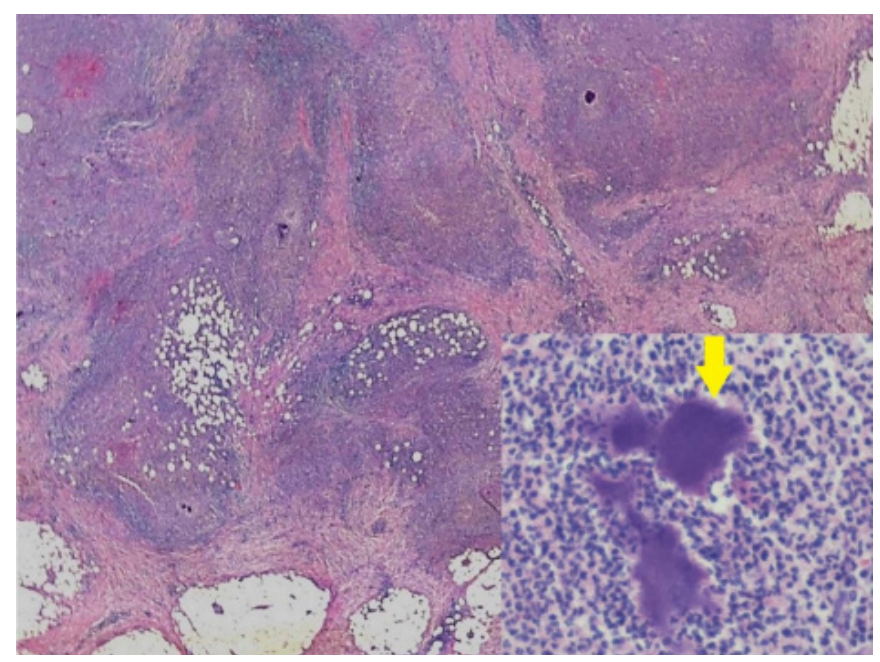

Fig. 4. Histopathological examination of the microscopic findings (H\&E stain). Diffuse inflammatory cell infiltration with multiple abscesses and fibrosis was observed $(\times 20)$. An actinomycotic sulfur granule was visualized (inset, $\times 200$ ).

tinomycosis and included chronic active inflammation with multiple abscesses, dense fibrosis, multiple lymphoid aggregates and follicles, dense eosinophilic infiltration, and a few actinomycotic sulfur granules in the abscesses (Fig. 4). The patient was treated with intravenous antibiotics for 2 weeks followed by oral amoxicillin and clavulanate for 2 months.

The Institutional Review Board of Yeungnam University Medical Center approved this case report and waived the requirement for informed consent (YUMC 2019-08-083).

\section{DISCUSSION}

Actinomycosis was first reported in 1846 by Bradshaw, who described a patient with fistula formation between a right iliac fossa mass and the skin [3]. Actinomycosis commonly affects the oral cavity, the female genital tract, and the bronchial system. Therefore, actinomycosis is classified into cervicofacial, thoracic, and abdominopelvic types based on the site of involvement [4]. Abdominopelvic actinomycosis is one of the main clinical types and is commonly associated with less than 4-year history of IUD implantation [5].

The clinical symptoms of actinomycosis are characterized by the infection's contiguous spread, suppurative and granulomatous inflammation, and formation of multiple abscesses and sinus tracts, resulting in dense fibrosis and formation of a hard mass. Additionally, individuals with mucous membrane injury and immune deficiency are predisposed to actinomycosis. A. israelii (the main causative agent) becomes saprophytic under anaerobic conditions and penetrates the injured mucosa [6]. Invasion of host tissue precipitates the inflammatory response which can result in the formation of multiloculated abscesses and pseudotumors.
Cope [3] reported that actinomycosis is one of the most misdiagnosed conditions and that no disease is so commonly missed, even by skilled clinicians. The preoperative diagnosis is challenging, particularly in patients with pelvic actinomycosis, for the following reasons; unusual presentation, low index of clinical suspicion, and difficulty with confirming actinomycetes as the causative organisms [7]. For example, actinomycosis of the gastrointestinal (GI) tract or the parietal peritoneum is often indistinguishable from GI tract carcinoma or carcinomatosis. Notably, actinomycosis involving multiple sites of the parietal peritoneum is rare [6]. Although CT is a useful diagnostic modality for visualization of actinomycotic lesions, it cannot conclusively establish the diagnosis [8]. Radiologic findings are nonspecific and quite similar to those observed in Crohn disease, intestinal tuberculosis, or sometimes an excavated malignant tumor [9]. Definitive diagnosis is necessarily based on isolation of actinomycetes from pus or histological examination of a suspicious mass. CT-guided aspiration with core needle biopsy is a useful diagnostic tool in cases of suspected actinomycosis. However, pus cultures rarely yield actinomycetes, so these lesions are commonly misdiagnosed preoperatively as malignant tumors. An accurate diagnosis is only possible postoperatively based on the histopathological examination of a surgically-excised specimen [10].

The lesions in our patient were initially suspected to be malignant tumors rather than inflammatory lesions for several reasons. There were no signs of an inflammatory response, such as fever or leukocytosis. Radiological findings on CT and MRI were consistent with multiple peritoneal masses, suggestive of malignant tumors rather than inflammatory abscesses. In addition, the CA125 laboratory value was elevated in our patient.

Although percutaneous needle biopsy was an option for tissue confirmation in the present case, we decided against it because the small tissues obtained by needle biopsy cannot provide an accurate diagnosis, especially in cases of multiple huge masses similar to our case. Furthermore, surgical biopsy using laparoscopic exploration was not possible because of a huge mass in the peritoneal and pelvic cavity. We, therefore, performed exploratory laparotomy.

Treatment of abdominopelvic actinomycosis depends on the extent of the disease and the patient's condition. Surgical resection is useful for definitive diagnosis and also as a therapeutic strategy for removal of infected tissue [11]. Antibiotic therapy alone can be considered in patients in whom actinomycosis can be conclusively diagnosed preoperatively. In our case, multiple intra-abdominal abscesses were identified, and carcinomatosis could not be ruled out. Therefore, surgical removal was performed, and actinomycosis was confirmed. Due to inflammatory reactive fibrosis and the risk of relapse, long-term antibiotic administration combined with surgical resection is warranted to ensure complete recovery from actinomycosis. Notably, Actinomyces spp. are usually susceptible to beta-lactams, particularly penicillin G. Clindamycin and tetracycline are useful alternatives in patients 
with adverse effects including penicillin allergy. The standard duration of antibiotic administration is unclear. In contrast to the previously recommended therapy of 6 to 12 months, short-term treatment over 2 to 6 weeks combined with surgical removal of lesions is known to be a successful treatment option [12].

We report a rare case of disseminated abdominopelvic actinomycosis mimicking carcinomatosis. Actinomycosis should be considered within the differential diagnosis for patients presenting with multiple abdominopelvic masses, radiological evidence of abscesses, and a history of long-term IUD implantation.

\section{CONFLICT OF INTEREST}

No potential conflict of interest relevant to this article was reported.

\section{REFERENCES}

1. Wagenlehner FM, Mohren B, Naber KG, Männl HF. Abdominal actinomycosis. Clin Microbiol Infect 2003;9:881-5.

2. Yegüez JF, Martinez SA, Sands LR, Hellinger MD. Pelvic actinomycosis presenting as malignant large bowel obstruction: a case report and a review of the literature. Am Surg 2000;66:85-90.

3. Cope VZ. Visceral actinomycosis. Br Med J 1949;2:1311-6.

4. Sung HY, Lee IS, Kim SI, Jung SE, Kim SW, Kim SY, et al. Clinical features of abdominal actinomycosis: a 15-year experience of a single institute. J Korean Med Sci 2011;26:932-7.

5. Nakahira ES, Maximiano LF, Lima FR, Ussami EY. Abdominal and pelvic actinomycosis due to longstanding intrauterine device: a slow and devastating infection. Autops Case Rep 2017;7:43-7.

6. Hildyard CA, Gallacher NJ, Macklin PS. Abdominopelvic actinomycosis mimicking disseminated peritoneal carcinomatosis. BMJ Case Rep 2013;2013:bcr2013201128.

7. Bae TS, Bae JD, Kim SO, Lee MS, Jung KH, Jung BW. Three cases of abdominal actinomycosis. J Korean Surg Soc 2000;59:414-9.

8. Ha HK, Lee HJ, Kim H, Ro HJ, Park YH, Cha SJ, et al. Abdominal actinomycosis: CT findings in 10 patients. AJR Am J Roentgenol 1993;161:791-4.

9. Triantopoulou C, Van der Molen A, Van Es AC, Giannila M. Abdominopelvic actinomycosis: spectrum of imaging findings and common mimickers. Acta Radiol Short Rep 2014;3:2047981614524570.

10. Pusiol T, Morichetti D, Pedrazzani C, Ricci F. Abdominal-pelvic actinomycosis mimicking malignant neoplasm. Infect Dis Obstet Gynecol 2011;2011:747059.

11. Huang CJ, Huang TJ, Hsieh JS. Pseudo-colonic carcinoma caused by abdominal actinomycosis: report of two cases. Int J Colorectal Dis 2004;19:283-6.

12. Wong VK, Turmezei TD, Weston VC. Actinomycosis. BMJ 2011; 343:d6099. 